\title{
Caractéristiques phonétiques de l'environnement phonétique des disfluences typiques du bégaiement : le cas de locuteurs français
}

\author{
Dodji Gbedahou ${ }^{1, *}$, et Fabrice Hirsch ${ }^{1}$ \\ 1Praxiling UMR 5267 CNRS, Université Paul-Valéry Montpellier 3, 34090 Montpellier, France
}

\begin{abstract}
Résumé. Cette étude s'inscrit dans la continuité de nos travaux antérieurs portant sur les disfluences typiques du bégaiement. En effet, les recherches menées sur la nature des sons disfluents en parole spontané et en description d'images ont révélé que ce sont essentiellement les voyelles antérieures, les voyelles de grande aperture et les voyelles nasales qui posent le plus de problème aux personnes qui bégaient (désormais $\mathrm{PQB}$ ) en français. En ce qui concerne les consonnes, les sujets testés présentaient des disfluences significativement plus importantes sur les consonnes nonvoisées et les post-alvéolaires. Cette nouvelle étude vient donc compléter cette dernière en abordant l'environnement phonétique des sons disfluents. L'objectif de ce travail est de définir les traits articulatoires des sons qui composent l'environnement phonétique des phonèmes ayant été bégayés. Pour y parvenir nous avons utilisé des enregistrements audios réalisés auprès de locuteurs bègues francophones du Togo pendant une activité de description d'images. Les résultats mettent en évidence que les consonnes qui viennent avant et après une consonne disfluente ne sont pas forcément de même nature. Certaines catégories de sons augmenteraient le risque de bégaiement s'ils se placent avant ou après une consonne. Mots clés : Bégaiement, parole, phonétique
\end{abstract}

\begin{abstract}
Phonetic characteristics of the environment of the typical disfluencies of stuttering: the case of french speakers. This study is a continuation of our previous work on the typical disfluencies of stuttering. Indeed, research on the nature of spontaneous speech disfluent sounds has revealed that it is mainly the anterior vowels, large aperture vowels and nasal vowels that pose the most problems to people who stutter (PWS) in French. For consonants, subjects exhibited significantly greater disfluences on unseen unvoiced consonants and post-alveolars. This new study complements the latter and have to analyze the phonetic environment of disfluent sounds. The main of this study is to define the articulatory features of the sounds that make up the phonetic environment of the phonemes that have been stuttered. To achieve this, we used audio recordings made in Togo from French speakers during a picture's description. The results show that consonant that come before and after a
\end{abstract}

* Corresponding author : dodji.gbedahou@univ-montp3.fr 
disfluent consonant are not necessarily of the same nature. Some categories of sounds would increase the risk of stuttering if they are placed before or after a consonant. Key words: Stuttering, speech, phonetic

\section{Introduction}

\subsection{Le bégaiement}

Par définition, le bégaiement est un trouble de la communication se traduisant mécaniquement par une forte présence de disfluences qui altèrent le rythme de la parole. Le bégaiement développemental, objet de notre recherche, touche entre 0,7 et $1 \%$ (Yairi \& Ambrose, 2013 ; Piérart, 2011).

Cela étant, plusieurs théories étiologiques s'essayent à expliquer ce trouble. Les tendances actuelles s'orientent vers une combinaison de facteurs parmi lesquels les aspects neurologiques (De Nil et al., 2008 ; Connally et al., 2014) et génétiques (Drayna \& Kang, 2011) sont les plus souvent avancés.

Afin de mieux comprendre les mécanismes du bégaiement, bon nombre d'études ont mis en évidence les traits phonétiques des phones qui posent le plus problèmes aux PQB. Néanmoins la plupart de ces études portent sur les locuteurs anglophones. Parmi ces travaux, une ancienne étude de Wingate (1979) relève que les syllabes accentuées sont les plus bégayées. D'autres études, comme celles de Jayaram (1983) et de Blomgren (2012), montrent des difficultés plus importantes sur les consonnes non-voisées. En fonction du lieu d'articulation et du mode articulatoire, ce sont essentiellement les labio-dentales (Blomgren, 2012) et les constrictives (Jayaram, 1983) qui sont les consonnes les plus disfluentes. En ce qui concerne les groupes consonantiques, ceux placés en début de mot sont les plus disfluents (Wolk, 2000).

Plus récemment, l'étude de Didirkova (2016), qui était menée auprès de locuteurs slovacophones et des locuteurs francophones sont venus compléter ces résultats. Celle-ci confirme d'une part les travaux les travaux de Jayaram (1983) et Blomgren (2012) quant aux taux de disfluence en fonction du voisement : les consonnes non-voisées seraient plus souvent disfluentes que leurs homologues voisées. D'autre part, Didirkova (2016) met également en évidence que, dans les deux langues (slovaque et français), ce sont les occlusives qui sont les consonnes les plus disfluentes.

Un récente étude (Gbedahou et al., 2019), se fondant sur les enregistrements de locuteurs francophones du Togo, s'est donnée pour objectif de définir les traits articulatoires des phones qui posent le plus de difficultés aux PQB.

En ce qui concerne les voyelles, ce sont les antérieures, celles de grande aperture et les nasales qui présentent les taux de disfluences les plus importants.

Quant aux consonnes, elles sont sujettes à davantage de disfluences sur les postalvéolaires et les non-voisées. Selon le mode articulatoire, il s'avère que ce sont les occlusives qui sont les plus disfluentes.

Comme le souligne ces résultats, certains traits phonétiques semblent être porteurs de davantage de disfluences que ce soit en anglais, en français ou en slovaque.

Mais la question est de savoir si les disfluences interviennent sur certains traits spécifiques ou si c'est la combinaison de deux traits côte à côte qui est responsable de l'émergence d'un bégayage. C'est sur cette problématique que va porter le présent article. En d'autres termes, l'objectif de cette étude est d'analyser les traits phonétiques des sons précédant et suivant une disfluence.

Notre hypothèse est que la présence de certains traits phonétiques influencerait l'apparition de disfluences bègues. 
Pour mener à bien cette étude, nous avons réalisé des enregistrements audio auprès de locuteurs francophones du Togo présentant un bégaiement développemental.

\subsection{Situation linguistique du Togo}

La langue officielle du Togo est le français depuis le 27 avril 1960. Cette dernière a été instaurée par la Constitution lorsque le Togo accède à l'Indépendance. Plusieurs raisons justifiaient ce choix.

Il s'agit dans un premier temps d'éviter les rivalités entre les populations du Nord et celles du Sud qui ne parlent pas les mêmes langues. En conséquence, prendre l'une des langues aurait probablement renforcer ces rivalités. De plus, selon Anzorgue (1998), le français était lors des indépendances la langue dont les locuteurs étaient les plus nombreux. En effet, 250000 togolais parlaient le français sur une population de 1102967 habitants.

En 2010, la proportion de locuteurs togolais sachant lire, écrire et comprendre le français est estimé à 34,37\% (RGPH, 2010).

Actuellement, le Togo compte environ 47 langues et dialectes. Ces différentes langues appartiennent toutes à la famille dite Niger-Congo. Le groupe Niger-Congo regroupe la totalité des langues de l'Afrique sub-saharienne en dehors des parlers tchadiques, nilosahariennes et khoi-san (Denis Creissels, 1989). Au sein de cette famille, il existe huit catégories :

Mandé ;

Kordfanien ;

Atlantique ;

Gur ;

Kru ;

Kwa ;

Benue-Congo ;

Admawa-Oubanguien.

Tableau 1. Les différentes langues du Togo

\begin{tabular}{|c|c|c|c|}
\hline Familles & Sous & pes & Langues \\
\hline \multirow{5}{*}{ Kwa } & \multirow{2}{*}{$\begin{array}{l}\text { Gbe et } \mathrm{Ga} \\
\text { adangbe }\end{array}$} & Gbe & $\begin{array}{l}\text { Adjagbe, evegbe, Fongbe, } \\
\text { gengbe,plagbe et Pedagbe }\end{array}$ \\
\hline & & Ga-adangbe & Adangbe \\
\hline & \multirow{2}{*}{ LVM3 } & $\mathrm{Ka}$ & Igo, ikposso,kebu \\
\hline & & $\mathrm{Na}$ & Anii, gidere \\
\hline & \multicolumn{2}{|c|}{ Tano } & Anufo,ginyanga \\
\hline \multirow{6}{*}{ Gur } & Oti-volta & Gourma & $\begin{array}{l}\text { Akaselem, gulmancema, } \\
\text { konkonba, migangam, } \\
\text { moba,ncam }\end{array}$ \\
\hline & & Occidental & Kussal, mampruli, moore \\
\hline & & Oriental & Biali, ditammari, mbelme \\
\hline & & $\begin{array}{l}\text { Yom- } \\
\text { Nawdm }\end{array}$ & Nawdm \\
\hline & & Miyobe & Miyobe \\
\hline & Gurunsi & & $\begin{array}{c}\text { Bago-kusuntu, delo, kabiyè, } \\
\text { lama, lukpa, tem }\end{array}$ \\
\hline $\begin{array}{l}\text { Benue- } \\
\text { Congo }\end{array}$ & \multicolumn{2}{|c|}{ Yoruba } & Ife, kambole, \\
\hline
\end{tabular}




\begin{tabular}{|c|c|c|}
\hline $\begin{array}{c}\text { Ouest- } \\
\text { Atlantique }\end{array}$ & Fulfude & Fulfulde \\
\hline Mande & Sud-Oriental & Bisa \\
\hline
\end{tabular}

Les langues et dialectes du Togo sont de deux types : les langues kwa et les langues Gur (tableau 1). Les langues Kwa se situent dans la partie méridionale du pays alors que les langues Gur s'étalent dans les régions septentrionales. Parmi ces langues, deux portent le statut de langues nationales : l'éwé, parlé en grande partie eu Sud et le Kabyè plus répandu dans le nord du pays. Néanmoins, la première langue véhiculaire du Togo est le mina.

\section{Matériel et Méthode}

\subsection{Population d'étude}

Les sujets ayant fait l'objet de cette étude sont au nombre de 11 et sont âgés de 21 à 43 ans. Notons que cette population est composée de trois femmes et huit hommes. L'âge moyen de cette population est de 27,181 ans avec un écart-type de 6,321 ans (Min = 20, Max=43). Les sévérités de bégaiement à l'épreuve de description d'images séquentielles varient de $5,3 \%$ à 23,56\% (Moyenne $=10,494$. ET=6,415) en mina. En revanche, en français, elles vont de 9,31\% à 28,04\% (Moyenne $=15,81$. ET=7,61). Quant au niveau socio-professionnel de cette population, il est constitué de cinq étudiants, deux ouvriers, trois cadres et une personne en recherche d'emploi (tableau 2).

Les enregistrements ont été effectués au moyen d'un enregistreurs audio de la marque Marantz PMD 561. Un fichier de type wav (44100 hz, 16 bit) a été obtenu.

Notons que les enregistrements ont été effectués dans un local calme au sein de l'école nationale des Auxiliaires médicaux de Lomé au Togo.

Les sujets testés ont été soumis à un exercice de description d'images séquentielles (Fig.1). Une série d'images racontant une histoire était montrée aux locuteurs qui avaient pour tâches de les mémoriser, puis de raconter cette histoire de mémoire. Préalablement, ils ont donné leur accord en remplissant un document de consentement. 

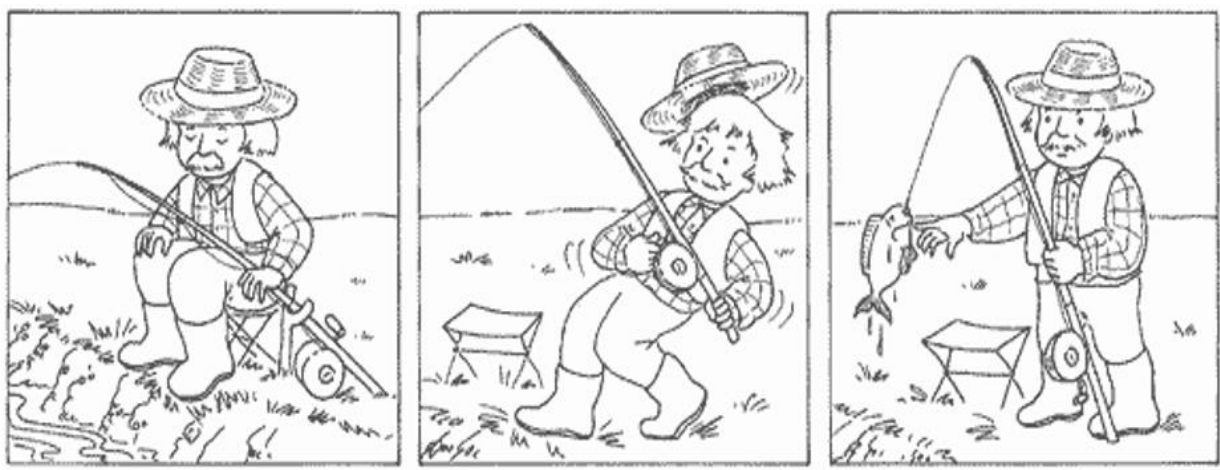

Fig.1. Description d'images séquentielles

Pour chaque sujet, la sévérité du bégaiement a été calculée en faisant le rapport entre le nombre de total de disfluences et le nombre de mot produit (Tableau 2).

Tableau 2. Sujet ayant participé à l'étude en fonction de l'âge, de la profession, et de la sévérité du bégaiement

\begin{tabular}{|c|c|c|c|}
\hline Locuteur & Age & Profession & Sévérité du bégaiement \\
\hline A & 27 & Étudiant & Très sévère \\
\hline B & 24 & Ouvrier & Sévère \\
\hline C & 20 & Étudiant & Modéré-Sévère \\
\hline D & 24 & Étudiant & Modéré-Sévère \\
\hline E & 26 & Ouvrier & Sévère \\
\hline F & 34 & Cadre & Modéré-Sévère \\
\hline G & 27 & Chômage & Très sévère \\
\hline H & 22 & Étudiante & Sévère \\
\hline I & 43 & Cadre & Modéré-sévère \\
\hline J & 26 & Étudiant & Modéré-sévère \\
\hline K & 26 & Cadre & Modéré-sévère \\
\hline
\end{tabular}

\subsection{Analyse et traitement des données}

Les fichiers audios ont été annotés sous Phon. L'annotation a été réalisée suivant deux lignes : une transcription lexicale puis une transcription basée sur l'Alphabet Phonétique International (API).

Tous les sons du corpus ont été ensuite répertoriés dans Excel puis le traitement statistique a été effectué sous le logiciel R studio. Notons que pour tous les sons disfluents 
insérés dans le fichier Excel, les caractéristiques des phonèmes avant et après ont été pris en compte. Pour les voyelles nous avons retenu le lieu d'articulation et l'aperture. Quant aux consonnes, les observations ont porté sur le lieu d'articulation, le mode articulatoire et le voisement. Nous avons observé les sons qui sont situés avant et après les consonnes bégayées.

\section{Résultats}

Ces résultats présenteront les sons qui précèdent et suivent les consonnes disfluentes. Dans chaque configuration, seront abordées les consonnes puis les voyelles.

\subsection{Répartition des consonnes qui précèdent une consonne disfluente}

\subsubsection{En fonction du lieu d'articulation}

Avant les consonnes disfluentes, nous avons répertorié :

- $22.581 \%$ de consonnes alvéo-dentales ;

- 9.677\% de consonnes alvéolaires ;

- $19.355 \%$ de consonnes bilabiales ;

- 35.484\% de consonnes uvulaires ;

- Et $12.903 \%$ de consonnes vélaires.

Comme on peut le noter, les consonnes uvulaires sont significativement présentes dans cette configuration, comparée aux alvéolaires.

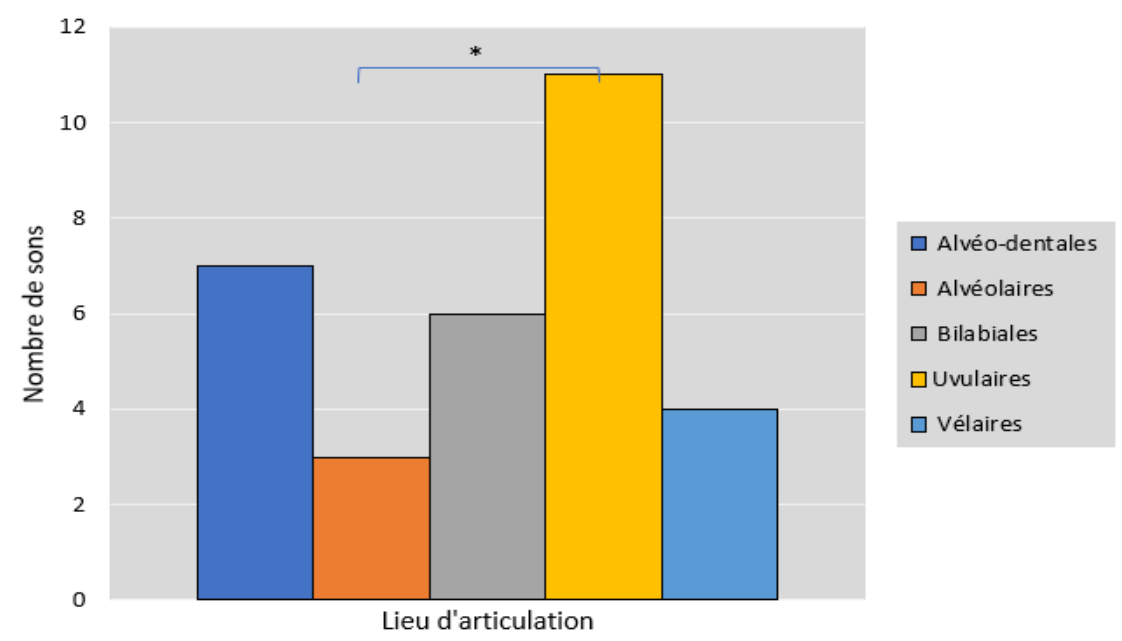

Fig. 2. Répartition des consonnes qui précèdent une consonne disfluente en fonction du lieu d'articulation, $\mathrm{p}<0.05$

\subsubsection{En fonction du mode articulatoire}


Le corpus analysé permet de noter un pourcentage plus important d'occlusives bégayées. En effet, les occlusives représentent $55 \%$ contre $42 \%$ pour les fricatives et $3 \%$ pour les spirantes.

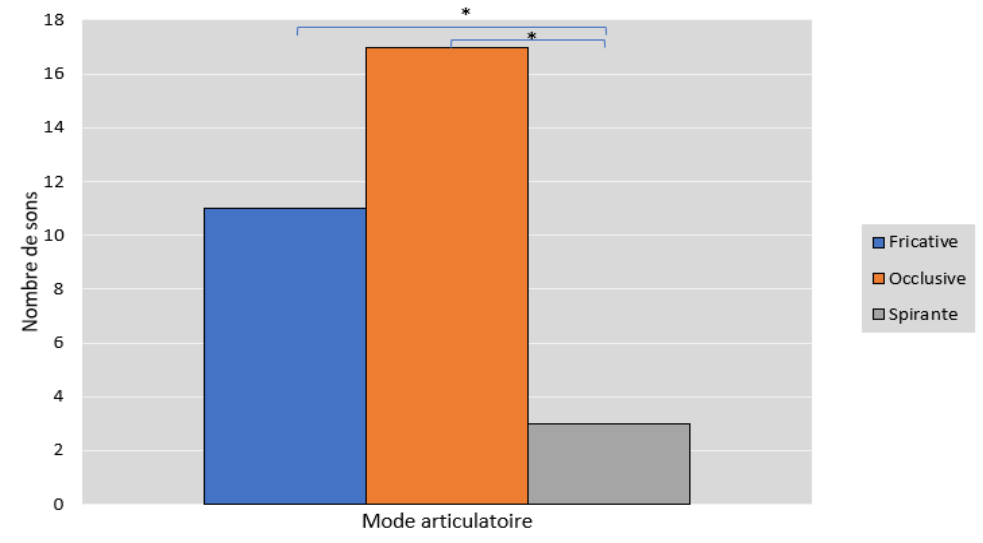

Fig.3. Répartition des consonnes qui précèdent une consonne disfluente en fonction du mode articulatoire, $\mathrm{p}<0.05$

En faisant une comparaison au moyen de Khi 2, on s'aperçoit d'une différence significative au sein de la répartition. En effet, l'on constate que les occlusives et les fricatives sont significativement plus importantes que les spirantes. Qu'en est-il de la répartition suivant le voisement?

\subsubsection{En fonction du voisement}

Dans cette comparaison, nous avons analysé les pourcentages des consonnes qui viennent avant les consonnes disfluentes. On s'apercoit dans cette analyse une proportion significativement plus importante de consonnes voisées. Ces phonèmes représentent 83.871 $\%$ de la répartition.

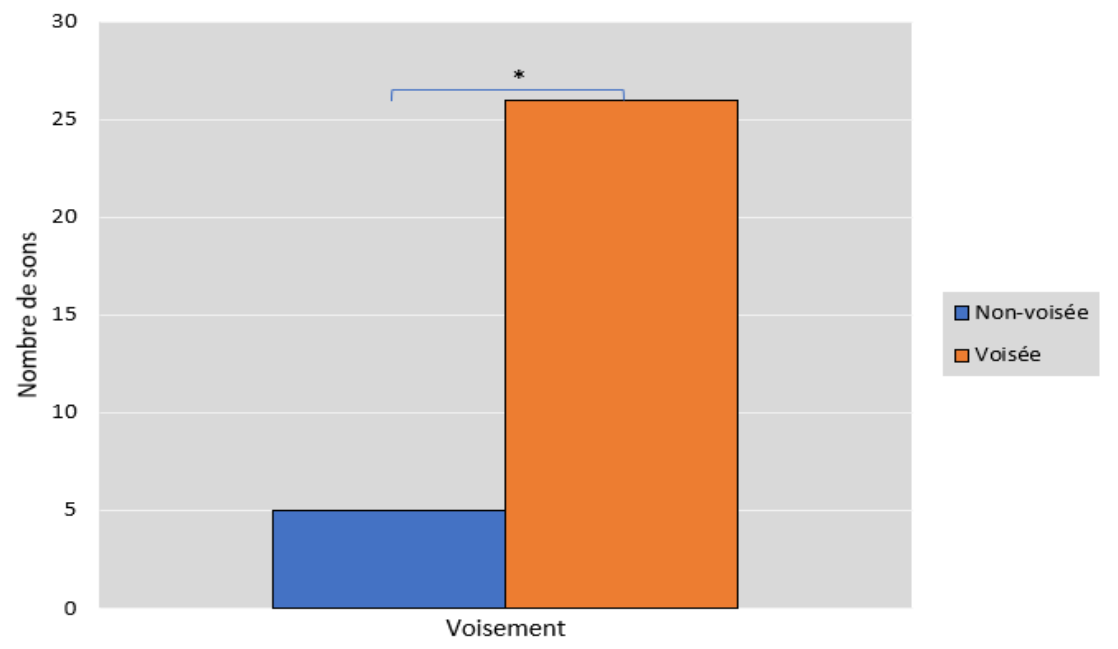

Fig.4. Répartition des consonnes qui précèdent une consonne disfluente en fonction du voisement, $\mathrm{p}<0.05$ 
Dans le prochain chapitre, nous étudierons les voyelles qui précèdent une consonne disfluente en langage spontané.

\subsection{Répartition des voyelles qui précèdent une consonne disfluente}

Nous avons enregistré dans ce corpus 143 voyelles placées avant une consonne bégayée. Nous étudierons les répartitions selon le lieu d'articulation et l'aperture des voyelles.

\subsubsection{En fonction du lieu d'articulation}

Selon le lieu d'articulation, les voyelles qui précèdent une consonne disfluente se répartissent de la manière suivante :

- 110 voyelles antérieures $(76,923 \%)$

- 20 voyelles centrales $(13,986 \%)$

- 13 voyelles postérieures $(9,091 \%)$

Comme l'illustre cette répartition, les voyelles antérieures sont majoritaires. En revanche on note une présence beaucoup moins importante des voyelles centrales et des voyelles postérieures.

En faisant une comparaison de manière générale, on s'aperçoit qu'il existe une différence au sein de la répartition (Khi2; $<<0.05)$

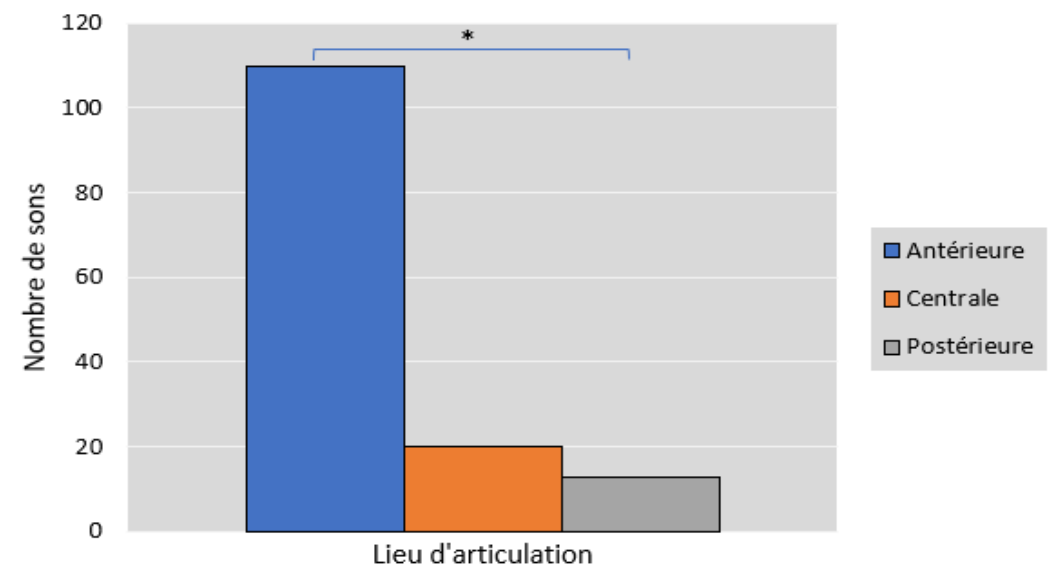

Fig.5. Répartition des voyelles qui précèdent une consonne disfluente en fonction du lieu d'articulation, $\mathrm{p}<0.05$

\subsubsection{En fonction de l'aperture}

L'étude portant sur l'aperture des voyelles qui précèdent une consonne disfluente permet de relever :

-24 voyelles de grande aperture, soit $17 \%$;

- 38 voyelles d'aperture mi-fermée, soit $27 \%$; 
- 45 voyelles d'aperture mi-ouverte soit $31 \%$;

- 20 voyelles d'aperture moyenne soit $14 \%$;

-Et 16 voyelles de petite aperture pour un pourcentage de $11 \%$.

On retiendra de cette distribution qu'il existe avant les consonnes disfluentes un pourcentage plus important de voyelles mi-ouvertes et mi-fermées.

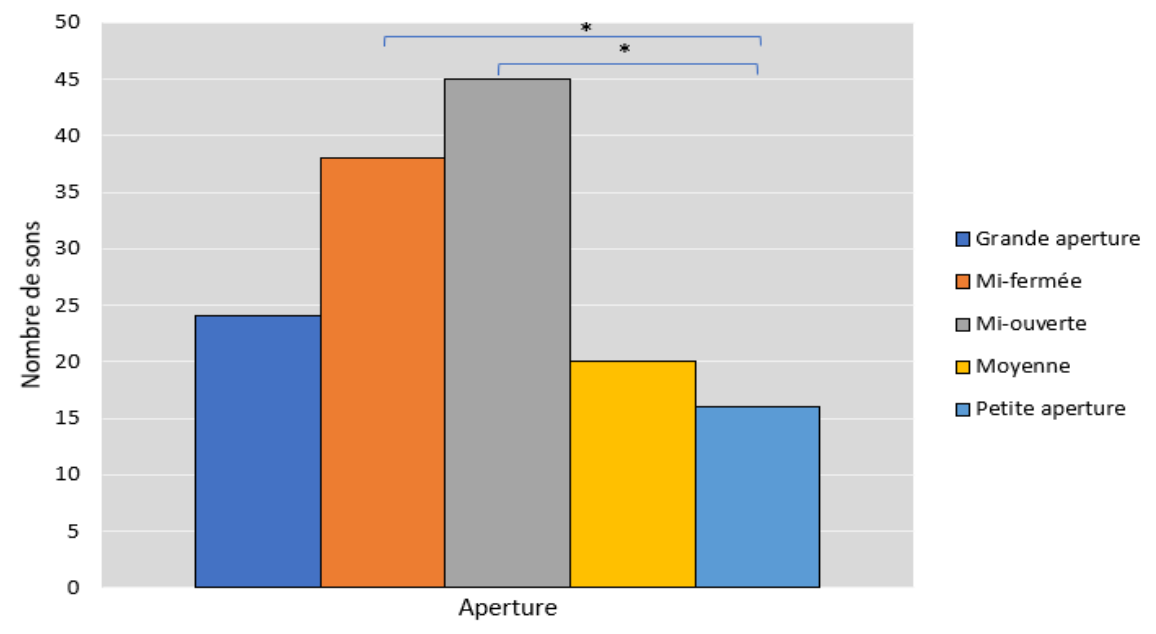

Fig.6. Répartition des voyelles qui précèdent une consonne disfluente en fonction de l'aperture, $\mathrm{p}<0.05$

Nous allons maintenant étudier la nature des sons qui suivent les phonèmes disfluents dans la chaine parlée.

\subsection{Répartition des consonnes qui suivent une consonne disfluente}

\subsubsection{En fonction du lieu d'articulation}

Comme le montre la figure 7 , six catégories de sons ont été observées : les alvéodentales, les alvéolaires, les bilabiales, les labio-dentales, les uvulaires et les vélaires.

Les alvéolaires représentent $33.33 \%$ des consonnes situées après une disfluence et sont significativement plus présentes que les alvéo-dentales, les bilabiales et les vélaires. 


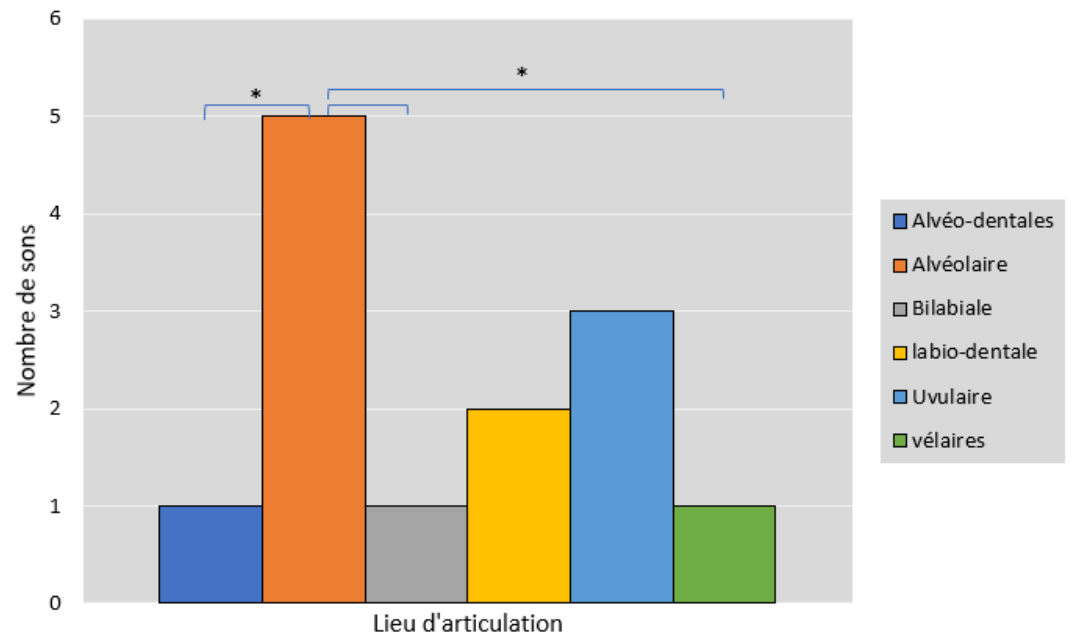

Fig. 7. Répartition des consonnes qui suivent une consonne disfluente en fonction du lieu d'articulation, $\mathrm{P}<0.05$

\subsubsection{En fonction du mode articulatoire}

L'étude portant sur le mode articulatoire des consonnes qui suivent une consonne disfluente en français révèle que les spirantes sont les plus présentes après une disfluence. Néanmoins les statistiques ne mettent pas en évidence une différence significative.

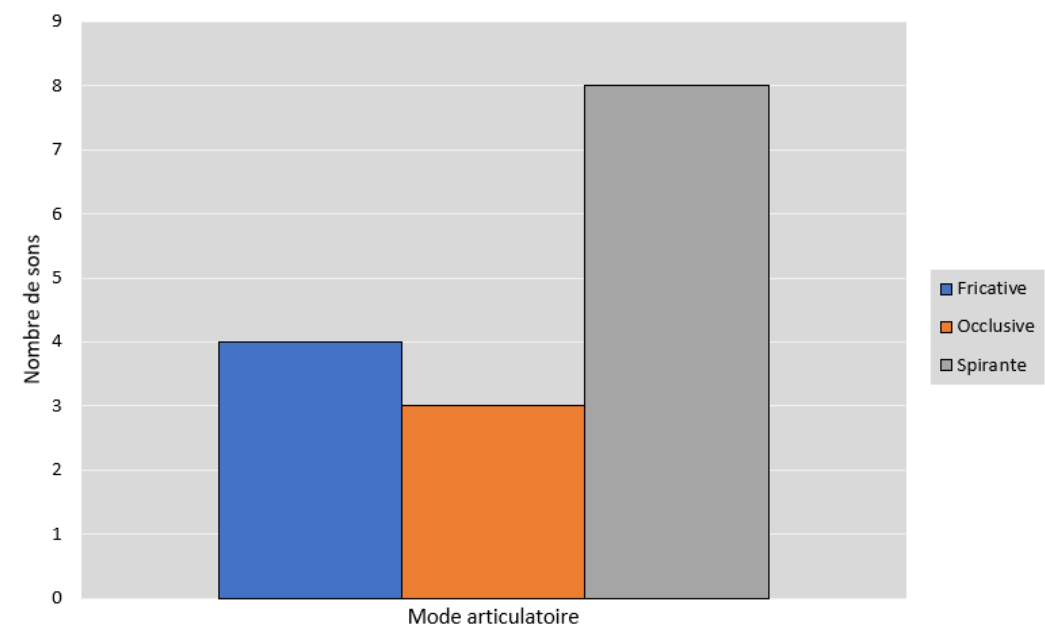

Fig.8. Répartition des consonnes qui suivent une consonne disfluente en fonction du lieu d'articulation, $\mathrm{p}>0.05$

\subsubsection{En fonction du voisement}

Si l'on s'intéresse aux consonnes qui viennent après les consonnes disfluentes selon le voisement, ce sont encore une fois les consonnes voisées qui sont les plus présentes. Elles représentent dans la distribution $93,333 \%$ des cas. 


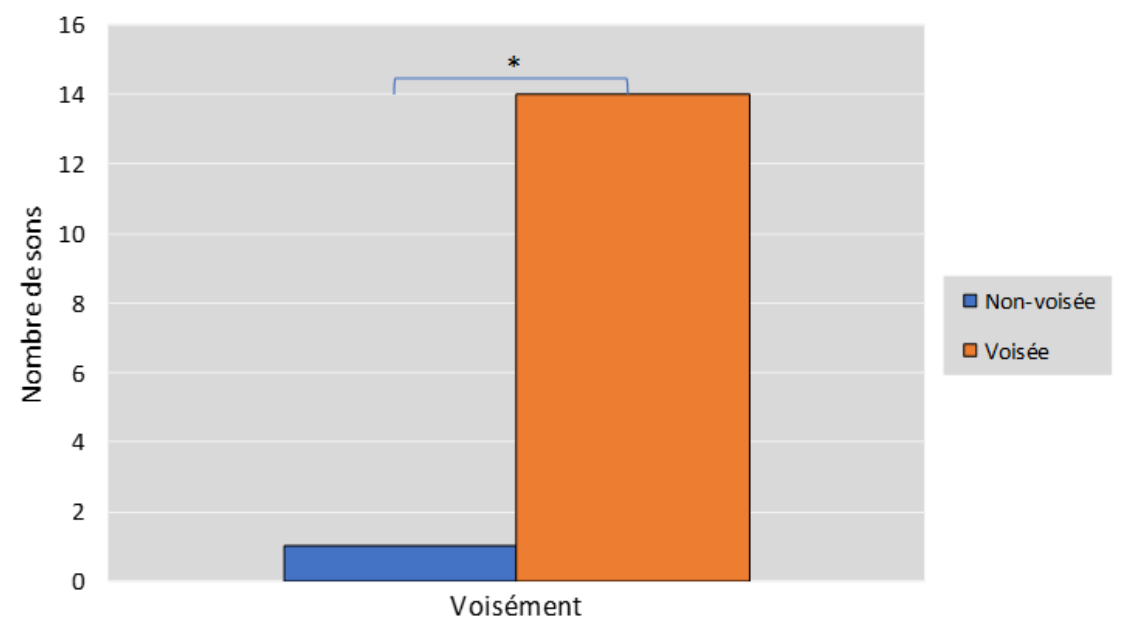

Fig.9. Répartition des consonnes qui suivent une voyelle disfluente en fonction du voisement, $\mathrm{p}<0.05$

\subsection{Répartition des voyelles qui suivent une consonne disfluente}

\subsubsection{En fonction du lieu d'articulation}

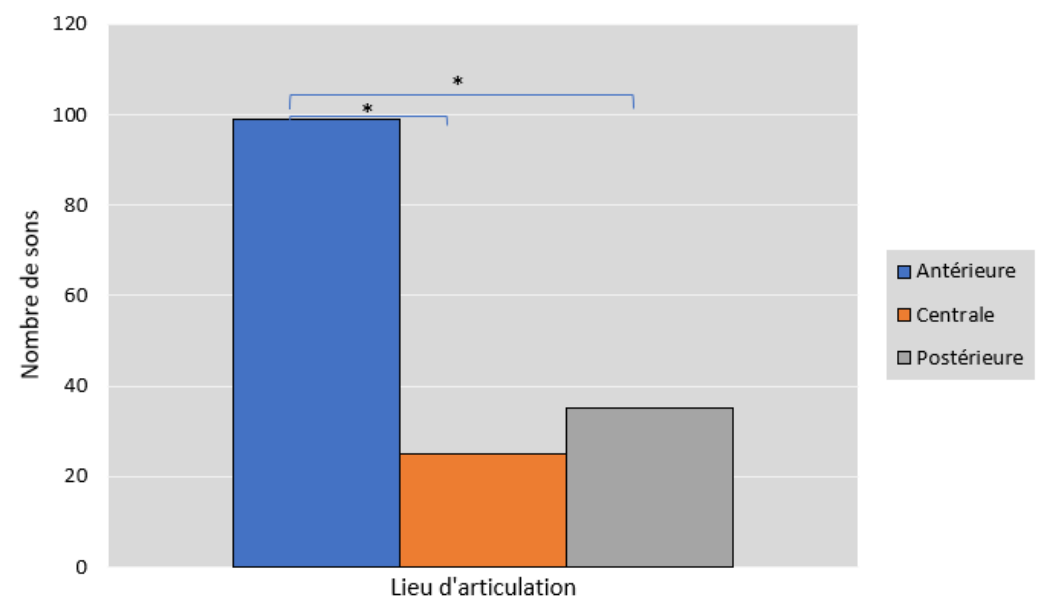

Fig.10. Répartition des voyelles qui suivent une consonne disfluente en fonction du lieu d'articulation, $\mathrm{p}<0.05$

L'objectif de cette comparaison est d'étudier l'effet du lieu d'articulation sur les voyelles qui suivent une consonne disfluente. On note une fréquence significativement plus importante des voyelles antérieures $(62.264 \%)$ comparée aux voyelles postérieures $(22.013$ $\%)$ et centrales $(15.723 \%)$.

\subsubsection{En fonction de l'aperture}




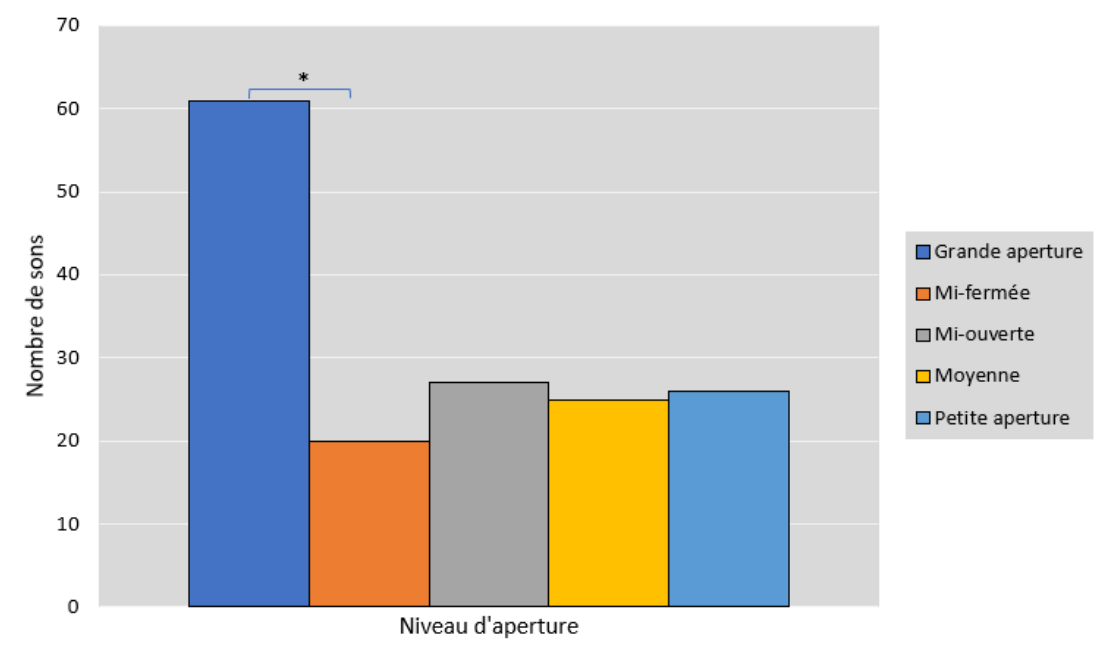

Fig.11. Répartition des voyelles qui suivent une consonne disfluente en fonction de l'aperture, $\mathrm{P}<0.05$

Nous avons observé l'effet de l'aperture sur les voyelles qui suivent une voyelle bégayée. On s'aperçoit dans cette étude qu'une voyelle serait d'autant plus disfluente si elle est suivie de voyelles de grande aperture. Ces voyelles représentent $38.365 \%$ des voyelles enregistrées contre $12.579 \%$ de voyelles mi-fermées, les voyelles les moins présentes.

\section{Discussion et conclusion}

Pour rappel, l'objectif de ce travail était d'étudier l'environnement phonétique des phones disfluents en français auprès des PQB. Notre hypothèse était que certains traits phonétiques pourraient être plus fréquents dans l'environnement phonétique des disfluences auprès des PQB.

Pour y parvenir nous avons utilisé les enregistrements audio réalisés auprès de 11 locuteurs qui bégaient du Togo âgés de 21 à 43 ans.

Les études se sont focalisées notamment sur les sons se situant avant et après les voyelles ou les consonnes disfluentes.

En ce qui concerne les consonnes disfluentes, elles présentent plus de risque d'être disfluentes si elles sont précédées de consonnes uvulaires, d'occlusives et de consonnes voisées. Aussi, si la voyelle précédant la consonne est antérieure ou mi-ouverte, la consonne présente un risque de disfluence plus important.

Quant aux sons qui suivent une consonne disfluente, une consonne présente plus de chance d'être disfluente si elle est suivie dans la chaine parlée de consonnes alvéolaires, de spirantes et de consonnes voisées. Les études révèlent par ailleurs que les voyelles qui suivent une consonne disfluente sont majoritairement les voyelles de grande aperture et les voyelles de grande aperture si on tient compte de l'aperture et les voyelles antérieures. 
De façon générale, on pourrait affirmer qu'une consonne présente plus de chance d'être disfluente si elle est placée avant ou après une voyelle antérieure. Ces voyelles, étant articulées à l'avant nécessitent une précision du point de vue articulatoire et cela pourrait justifier ces résultats. Ces voyelles sont d'ailleurs les plus disfluentes selon notre étude antérieure (Gbedahou et al, 2019)

Par ailleurs on note également que les consonnes voisées sont plus présentes dans l'environnement phonétique des consonnes disfluentes. Or, l'une de notre précédente étude (Gbedahou et al., 2019) a mis en évidence que les consonnes non-voisées présentaient le plus de disfluence. On pourrait penser que la succession consonnes voisées/non-voisées pourrait entrainer plus de bégayages. En effet, la transition entre un élément voisé et un autre qui ne l'est pas serait problématique et entrainerait plus de disfluences chez les PQB.

Suivant le lieu d'articulation et le mode des consonnes d'une part et l'aperture des voyelles d'autre part, les résultats diffèrent si le son se place avant ou après la consonne disfluente. En effet, les consonnes qui précèdent une consonne disfluente sont des uvulaires et des occlusives alors que lorsqu'on étudie les consonnes qui suivent une consonne disfluente, on compte davantage d'alvéo-dentales et l'on n'observe pas d'effet significatif selon le mode articulatoire. Pour finir, si ce sont les voyelles mi-ouvertes qui précèdent le plus les consonnes bégayées, on n'observe pas les mêmes résultats en étudiant les voyelles qui suivent une consonne disfluente. Celles-ci sont essentiellement constituées de voyelles de grande aperture.

Comme perceptive, nous envisageons étudier l'environnement phonétique des voyelles disfluentes, c'est-à-dire les types de sons qu'on observe avant et après une voyelle disfluente.

\section{Références bibliographiques}

Anzorge, Isabelle. 1998. Le français au Togo : une aventure ambiguë. Le français en Afrique : revue du réseau des observatoires du français contemporain en Afrique 12.

Blomgren, M. (2012). Do Speech Sound Characteristics Really Influence Stuttering Frequency ? In Proceedings of the 7th World Congress of Fluency Disorders.

Bole-Richard, Rémy. 1983. Systématique Phonologique et Grammaticale d'un Parler Ewe: Le GenMina Du Sud-Togo et Sud-Bénin. Paris: Editions L'Harmattan.

Connally, E. L., Ward, D., Howell, P., \& Watkins, K. E. (2014). Disrupted white matter in language and motor tracts in developmental stuttering. Brain and language, 131, 25-35.

De Nil, L.F., Beal, D.S., Lafaille, S.J., Kroll, R.M., Crawley, A.P., \& Gracco, V.L. (2008). The Effects of Simulated Stuttering and Prolonged Speech on the Neural Activation Patterns of Stuttering and Nonstuttering Adults. Brain and Language, 107(2):114-123.

Drayna, Dennis, and Changsoo Kang. 2011. "Genetic Approaches to Understanding the Causes of Stuttering." Journal of Neurodevelopmental Disorders 3(4): 374-80.

Gbedahou, Dodji \& Didirkova, Ivana \& Hirsch, Fabrice. (2019). Caractéristiques phonétiques des disfluences typiques du bégaiement dans un contexte bilingue : le cas des locuteurs français-mina.

Gblem-Poidi, Massanvi Honorine, and Laré Pierre Kantchoa. 2012. Les langues du Togo : état de la recherche et perspectives. Paris: Harmattan. 
Jayram, M. (1983). Phonetic Influences on Stuttering in Monolingual and Bilingual Stutterers. Journal of Communication Disorders, 16(4):287-197.

Piérart, B. (2011). Les bégaiements de l'adulte (Vol. 5). Editions Mardaga.

Wolk, L., Blomgren, M. \& Smith, A. (2000). The Frequency of Simultaneous Disfluency and Phonological Errors in Children: A Preliminary Investigation. Journal of Fluency Disorders, 25:269-281.

Yairi, E., \& Ambrose, N. (2013). Epidemiology of stuttering: 21st century advances. Journal of fluency disorders, 38(2), 66-87. 